\title{
Hochschulen sind frei - aber sind sie auch gut? Zur Evaluation von Lehre und Forschung mit dem Schwerpunkt Fachhochschulen
}

\author{
Peter Gadow
}

\section{Allgemeine Evaluationssituation}

Die Freiheit von Lehre und Forschung ist ein unbestritten hohes Gut, das gerade vor dem Hintergrund deutscher Geschichte zu schützen und zu pflegen ist. In der Hochschulpraxis fuihrt diese Unabhängigkeit aber auch oft zu negativen Begleiterscheinungen. Wer an einer Universität oder Fachhochschule studiert, kennt dies aus eigenem Erleben. Lehrpläne sind nur bedingt aufeinander abgestimmt, in manchen Fächern gibt es inhaltliche Überlagerungen, Schwerpunktfächer sind nur unzureichend präsent, mangelhafte Methodik bei guter Fachkompetenz führt gerade in den ersten Semestern zu Orientierungs- und Verständigungsproblemen usw. [1] Auf der anderen Seite kommen viele Studenten nur mit wenig konkreten Vorstellungen über ihr Studienfach an die Hochschulen und studieren anschließend relativ motivationslos vor sich hin. Man fuihlt sich überfordert und erkennt erst zu spät, dass man sich für ein falsches Studium entschieden hat. Hinzu kommt die Anonymität der „Massenhochschulen“, die den Eindruck der Orientierungslosigkeit verstärkt.

Ein dritter Aspekt ist in diesem Zusammenhang von Bedeutung. Auf der einen Seite wirft die Wirtschaft den Hochschulen vor, sie bilden praxisfern aus und betreiben Forschung im Elfenbeinturm, während auf der anderen Seite Repräsentanten der Wissenschaft der Wirtschaftspraxis kurzsichtigen Aktionismus unterstellen. [2]

\section{Evaluationssituation}

Ziel der Evaluation von Lehre und Forschung an den Hochschulen muss es daher sein, Studiengänge besser zu organisieren und sie entsprechend den definierten Studiumszielen und Ausbildungsprofilen praxisnah auszurichten. Dabei sollte eine hohe Qualität von Lehrveranstaltungen und Forschungsleistungen sichergestellt werden.

Ein gutes Gelingen des Studiums setzt seitens der Studierenden eine qualifizierte Vorbildung voraus sowie eine bewusste Entscheidung fuir das Studienfach. Anforderungen an die Hochschullehrer sind insbesondere eine hohe Motivation, Kompetenz und ein großes Engagement, um Studenten berufsnah ausbilden zu können. Um diese Kriterien ermitteln zu können, sind in der Vergangenheit in der deutschen Hochschullandschaft verschiedene Versuche unternommen worden mit Evaluationsverfahren Bewertungsgrundlagen zu schaffen. So gibt es z. B. einen sogenannten Evalua- tionsverbund norddeutscher Hochschulen, der aus fünf Universitäten besteht.

Niedersachsen hat eine Agentur gegründet, die sich mit der Evaluation der Lehre an niedersächsischen Hochschulen beschäftigt, und auch die FHW Berlin erprobt ein geeignetes Instrumentarium zur Evaluation. [3] In Sachsen ist jüngst das „Studentische Evaluationsbüro Sachsen" (SES) eingerichtet worden, mit dem die Studenten des Freistaates die Qualitätssicherung an den Hochschulen des Landes sicherstellen wollen. „Die Berichte des Evaluationsbüros sollen über die Situation von Lehre und Studium informieren und durch Hinweise auf methodische und didaktische Mängel in den einzelnen Fachbereichen öffentlichen Druck auf die Hochschulen, aber auch auf die politische Verantwortung ausüben." Andererseits könnten gute Bewertungen der Studenten die Anziehungskraft einzelner Hochschulen vergrößern. [4]

\section{Bisherige Evaluationspraxis}

Die bislang praktizierten Formen der Evaluation sind zweigeteilt. Es wird eine interne und eine externe Evaluation unterschieden.

Die interne Evaluation sieht allgemein einen Selbstreport zu Studium und Lehre vor. Bei der externen Evaluation wird von einer Gutachtergruppe eine sogenannte Begehung der Hochschule vorgenommen, um anschließend auf der Grundlage des Selbstreportes eine Beurteilung und Verbesserungsvorschläge zur Gestaltung des Studiums abzugeben. [5]

Mit Hilfe dieser Evaluationsverfahren sollen

- die Leistungen der Fachbereiche umfassend dokumentiert werden,

- ein Aufschluss über die Mittelverteilung erreicht werden,

- Maßstäbe für die aufgabenbezogene Bewertung der Qualität und

- Verfahren zur Qualitätssicherung auf Fach- und Fachbereichsebene entwickelt werden.

\section{Interne Evaluation}

Um diese Ziele zu erreichen, steht zunächst die interne Evaluation im Vordergrund. Sie soll Hinweise auf Stärken und Schwächen in der Lehre aus Sicht der Professoren, der wissenschaftlichen Mitarbeitern und der Studenten geben. 
„Die interne Evaluation sollte Korrekturen und Anpassungen innerhalb der Fächer im Hinblick auf die Anforderungen von Forschung, Lehre und Nachwuchsförderung einleiten oder erleichtern, aber auch dazu geeignet sein, neue Zielsetzungen inhaltlicher oder organisatorischer Art zu definieren und ihre Umsetzung zu fördern." [6]

Die Mittel, mit denen die interne Evaluation durchgeführt werden kann, sind vielfältig. Zu ihnen zählen z. B. Lehrberichte der Fachbereiche, Befragungen von Dozenten und Studenten, aber auch Absolventenbefragungen und Arbeitsmarktanalysen. Auf der Grundlage der so ermittelten Daten und Fakten kann ein Vergleich mit definierten Zielsetzungen der Hochschulen (Studienordnungen und Studienplänen) erfolgen. Die Ergebnisse sollten dann in einem Evaluationsbericht alle fünf Jahre zusammengefasst werden.

Nach Auffassung der Hochschulrektorenkonferenz umfasst der Evaluationsbericht das „Ziel des Studienganges und nimmt Stellung zur Akzeptanz der Hochschullehre, zu Analyse, Bewertung, Kritik und Vergleich von Leistungen in der Lehre." [7] Der Bericht ist in erster Linie-Rüickmeldung an die Lehrenden, die Studierenden, den Fachbereich und die Hochschulleitung. Da er sich nicht auf die Inhalte der Lehre bezieht und schon gar nicht auf handelnde Personen, kann er auch nicht als Beeinträchtigung von Lehre und Forschung interpretiert werden.

Sinn der internen Evaluation ist vorrangig die Erstellung einer kritischen Selbstbewertung im Hinblick auf selbstgestellte Ziele und Aufgaben der Fachbereiche. Gleichzeitig sind die Hintergründe für offensichtliche Fehlentwicklungen aufzuzeigen und Maßnahmen zur Zielerreichung zu entwickeln. Dazu zählt auch die Bewertung eigener Initiativen zur Verbesserung der Lehre und des Lehrerfolges sowie die Anpassung der Lehrinhalte an wissenschaftliche und berufliche Anforderungen.

\section{Externe Evaluation}

Die externe Evaluation soll die interne Evaluation auf ihre Wirksamkeit hin durch eine externe Expertengruppe kritisch prüfen. Darüber hinaus soll die externe Evaluation auch eine Grundlage schaffen, gleichartige Institutionen im Hochschulbereich fachbezogen zu vergleichen. Aus Sicht der Hochschulrektorenkonferenz sind die wichtigsten zu bewertenden Problemfelder:

- „Ziele des Studiums und ihre Umsetzung

- Aufbau und Struktur des Studienganges und der Lehrgebiete (Verhältnis von Vorlesungen zu Seminaren und Übungen, Grund- und Hauptstudium, Vertiefungsgebiete)

- Organisation von Lehre (Beseitigung von Kollisionen im Lehrveranstaltungsangebot auch im Hinblick auf die Studierbarkeit von Haupt- und Nebenfächern, Studienplänen und Prüfungsabläufen)

- Didaktische Fragen (Stand der Befragungsergebnisse, Verfügbarkeit von Lehrmaterialien in schriftlicher und anderer Form)

- Probleme beim Übergang von der Schule zur Hochschule und ihre Lösungen
- Probleme beim Übergang von der Hochschule in den Beruf und ihre Lösungen

- Allgemeine und Studienfachberatung

- Einbeziehung der Forschung in die Lehre

- Heranbildung des wissenschaftlichen Nachwuchses

- Vorkehrungen zur Sicherung der Qualität der Lehre“ [8]

Die externe Evaluation sollte durch eine Expertengruppe von ca. 5 bis 6 Personen erfolgen, die neben Wissenschaftlern auch Vertreter der Wirtschaft bzw. andere Absolventenabnehmer umfasst. Der mit der externen Evaluation verbundene Aufwand lässt einen längeren Untersuchungsabstand von ca. 10 Jahren sinnvoll erscheinen.

\section{Kritik der bisherigen Evaluation}

Einheitliche Messverfahren zu entwickeln, mit denen die Leistungsfähigkeit von Hochschulen ermittelt werden kann, ist sicher sinnvoll. Von den oben skizzierten Beispielen abgesehen steht die Evaluation von Lehre und Forschung in Deutschland im Vergleich zum angelsächsischen Bereich erst am Anfang.

Offensichtlich ist die Akzeptanz der Verfahren im Hochschulbereich umstritten. Dies hat viele Gründe. Welche Hochschule und welche politisch Verantwortlichen lassen sich schon gerne nachsagen, dass Lehre und Forschung an der einen oder anderen Universität oder Fachhochschule wenig effektiv oder praxisfern gestaltet werden?

Sind die Erhebungstechniken z. B. in Form von Fragebögen tatsächlich valide, oder ist der Manipulation hier nicht Tür und Tor geöffnet?

„Ein Ranking von Vorlesungen per Teilnehmerbefragung ist ein völlig untaugliches Mittel, die Qualität von Lehre nachzuweisen, und ist letztlich methodischer Unsinn. Es lässt sich nämlich zeigen, dass das studentische Urteil über Lehrveranstaltungen beinahe unabhängig von den Merkmalen bzw. der Qualität der Lehre ist, vielmehr hängen die Ergebnisse der Beurteilungen von den Lehrveranstaltungen $\mathrm{ab}$, von

- der Motivation der Vorlesungsteilnehmer,

- den Wahlmöglichkeiten, den persönlichen Interessen und Erwartungen,

- dem Studienverhalten und der Studienerfahrung, " [9]

- der Zufriedenheit der Studenten, die nicht identisch mit der Motivation sein muss.

Kann darüber hinaus sichergestellt werden, dass die Ergebnisse der internen und externen Evaluation auch einer breiten Öffentlichkeit zugänglich gemacht werden? Fast alle bisher erarbeiteten Berichte der Fächer zur Selbstevaluation, insbesondere nach dem Modell der internen und externen Evaluation, dienen vorrangig der internen selbstkritischen Auseinandersetzung mit Studium und Lehre. Konsequenterweise werden diese Berichte in der Regel nur fachintern veröffentlicht. [10] Ist weiter davon auszugehen, dass es bei unterschiedlichen Evaluationsmethoden uiberhaupt zu einer Vergleichbarkeit zwischen den diversen deutschen Hoch- 
schulen kommen kann? Bis heute gibt es, von wenigen Ausnahmen abgesehen, kaum Abstimmungen über die Zusammenstellung der Datengrundlage und das methodische Vorgehen. Die daraus folgenden Abweichungen in der Gewinnung und Aufbereitung der Ergebnisse lassen Vergleiche nur sehr eingeschränkt zu.

Vor diesem Hintergrund ist das aktuell beliebte Universitäts- oder Fachhochschulranking im „Stern“ oder „Focus“ mehr als fragwürdig, da wenig valide.

Und schließlich, wie sieht es mit der Umsetzung der Evaluationsergebnisse aus? Bislang gibt es offensichtlich wenig Bereitschaft, notwendige Konsequenzen zu ziehen. Exemplarisch heißt es dazu in einem Berliner Evaluationsbericht: „Das großangelegte Evaluationsprojekt an der TU droht nach zwei Jahren fleißigen Auftürmens von ebenfalls anonymisierten Datenbergen im Sande zu verlaufen, weil sich unter den Verantwortlichen niemand mehr findet, der über notwendige Schlussfolgerungen aus den Befragungsergebnissen sprechen möchte." [11]

Solange hinsichtlich dieser offenen Fragen keine Klarheit herrscht, bleibt Evaluation von Lehre und Forschung ein wünschenswertes und notwendiges aber umstrittenes Instrumentarium zur Qualitätssicherung an deutschen Hochschulen.

\section{Problem}

Um eine Evaluation zu optimieren, sollte man noch einmal versuchen, die Ziele einer Hochschule adäquat zu definieren. Hierzu ist es erforderlich, die im deutschsprachigen Gebiet vorliegende Trennung der Hochschulen in Universitäten und Fachhochschulen zu berücksichtigen. Die für die weiteren Betrachtungen ausreichende Differenzierung wird folgendermaßen gemacht:

- Universitäten bieten eine akademisch ausgerichtete Ausbildung an. Sie sind stark auf Grundlagen und Theorien eines Faches ausgerichtet.

- Fachhochschulen (im weiteren Hochschulen genannt) bieten eine wissenschaftlich praxisorientierte Ausbildung an. Hier werden die nach dem letzten Stand der wissenschaftlichen Erkenntnis und in die Praxis übernommenen Methoden und Verfahren für die Ausbildung eingesetzt.

Im Weiteren werden nur noch die Hochschulen betrachtet. Für die Universitäten müssten vergleichsweise ähnliche Überlegungen im Einzelnen angestellt werden. Hier soll der Versuch unternommen werden, Hochschulevaluation konsequent aus der Sicht des Marketings zu betrachten. Dies ist ein ungewohnter, aber sicher zu exakteren Evaluationsverfahren führender Ansatz. Die Kunden bzw. die Zielgruppen wären schwerpunktmäßig die Wirtschaft, Verwaltung und gesellschaftliche Institutionen. Universitäten und in noch höheren Maße Hochschulen werden zunehmend zu Dienstleistungsinstitutionen. Aus Marketingsicht wäre das eine Verschiebung von der Angebots- zur Nachfrageorientierung. Die Dienstleistung oder das Produkt wäre die optimale Ausbildung der Studenten. Die Kundenzufriedenheit mit der Dienstleistung oder dem
Produkt äußert sich in der Bereitschaft, die Dienstleistung - den ausgebildeten Studenten einer bestimmten Hochschule in einer bestimmten Fachrichtung sofort zu übernehmen und angemessen zu entlohnen. Selbstverständlich sind auch hier konjunkturelle und strukturrelevante Einflüsse zu berüicksichtigen. Als Beispiel sei nur die Situation der Ingenieure in Deutschland erwähnt. Der Bedarf der Maschinenbauingenieure, stellvertretend auch für andere Ingenieurrichtungen, war bis vor kurzem negativ. Somit wurde faktisch eine zu geringe Zahl von Maschinenbauingenieure ausgebildet. Der Bedarf zur Zeit läßt sich nicht mehr durch die Absolventen der vergangenen Jahre decken, so daß hier eine hohe Nachfrage herrscht und die Wirtschaft im Prinzip auch bei geringere Zufriedenheit jeden Absolventen nimmt.

Hier tut sich sofort eine neue Problematik auf. Der „Rohstoff " für das Produkt - der Absolvent mit seinem Diplom - ist der immatrikulierte Student am Anfang des Studiums mit seiner Vorausbildung und Grundeinstellung zu seiner gewählten Fachrichtung. Dieser Rohstoff ist nicht beliebig beschaffbar.

Aus Marketingsicht kann der Prozess der Ausbildung an einer Hochschule folgendermaßen betrachtet werden:

- Die Wertschöpfung (Ausbildungsprozess) an der

Hochschule muß optimal gestaltet werden. Dies geschieht durch geeignete Strukturen und die entsprechenden Professoren in der Lehre sowie eine flexible und optimale Hochschulstruktur und ein optimales Hochschulmanagement.

- Der Kunde, stellvertretend wird hier nur die Wirtschaft betrachtet, die als größter Kunde oder Abnehmer der Absolventen einer Hochschule im Allgemeinen auftritt, muss mit dem Produkt der Hochschule voll zufrieden sein und seine Probleme optimal, d. h. hier in kurzer Zeit (also ohne lange Einarbeitungszeiten und zusätzliche Ausbildungsanstrengungen) lösen können.

- Die Qualität der Dienstleistung (Ausbildung des Studenten) ist wesentlich von der Güite des Vorproduktes (Vorbildung und Grundeinstellung der zukünftigen Studenten) abhängig. Damit sind im einfacheren Falle z. B. gewisse Vorkenntnisse in Mathematik, Naturwissenschaften und Motivationen gemeint. Dies kann durch Brückenkurse oder andere geeignete Maßnahmen verbessert werden. Die Studieneinstellung kann generell durch geeignete Maßnahmen der Vorauswahl, Aufklärung und Public-Relations-Arbeit sowie eine Vorauswahl im begrenzten Maße gesteuert werden. Im entscheidendem Maße ist die Qualität der Dienstleistung natürlich gegeben durch das Vermögen des Lehrkörpers, moderne Lehrmethoden einzusetzen und eine hohe Bereitschaft, individuell auf Probleme der Studenten einzugehen. Als Maß für die Weiterbildung des Lehrkörpers und die Gewährleistung eines hohen praxisbezogenen Kenntnisstandes kann die Anzahl von Forschungsprojekten, der Teilnahme am Technologietransfer und die Einwerbung von Drittmittel dienen.

- Die Qualität der Hochschulstrukturen sowie das Hochschulmanagement, die Güte des „Vorproduktes“ 
Student, seines Wissens und seiner Motivation, die Qualifikation der Lehrmethoden, praxisbezogene Kenntnisstände und Projekte werden aber letzlich immer vom Kunden bewertet, indem er die Leistung und Brauchbarkeit seines neuen Mitarbeiters einschätzt. Die Evaluation der hochschulinternen Parameter ist folglich nur dann aussagekräftig, wenn sie mit der externen Kundenevaluation übereinstimmt.

\section{Grundlagen}

Aus der bisherige Betrachtung lässt sich eine Bestimmung der Evaluation gewinnen, die für die weiteren Überlegungen ausreicht. Sie ist sicher nicht vollständig, aber für unsere Problembetrachtung ausreichend.

Man kann unterscheiden zwischen einer

- externen und einer

- internen

Evaluation. Weiterhin ist eine weitere Unterteilung in

- objektiv und

- subjektiv

sinnvoll.

Wird zum Beispiel ein externes Unternehmen beauftragt, die Hochschule zu evaluieren, so wird es einmal versuchen, objektive Kennzahlen zu bilden oder zu übernehmen (z. B. absolute Zahlen von Studenten und andere statistische Zahlen) und sie mit anderen Hochschulen gleichen Typus zu vergleichen. Zum anderen wird es auch objektivierende sozio-psychologische Messungen (Befragungen) durchfuihren. D. h. es werden Personen, die in irgendeiner Weise mit den Hochschulen verbunden oder betroffen sind, befragt. Diese sogenannten Stakeholders können Mitglieder oder keine Mitglieder der Hochschule sein. Mit diesen Ergebnissen lässt sich ein Kundenurteil über eine Hochschule gewinnen bzw. ist mit anderen Hochschulen zu vergleichen. Dies kann man dann in Rankinglisten zusammenfassen.

Objektivierte hochschulinterne Messungen und Vergleiche können durchaus einen Sinn für die Verbesserung der Lehr- und Lerntätigkeit haben, sie eignen sich möglicherweise auch für einen Methodenvergleich der Hochschulen, aber auf keinen Fall sind sie für ein Ranking geeignet.

Die auf subjektive Einschätzungen (Zufriedenheitsgefühl) beruhende Rankinglisten, meist im Auftrage von Zeitschriften durchgefuihrt und dann veröffentlicht, haben im Allgemeinen keinen Wert. Sie sind nach wissenschaftlichen Kriterien nicht fundiert und besitzen keine kritische Interpretation der Ergebnisse. Dies kommt auch schon in den sehr unterschiedlichen Rankinglisten in den einzelnen Zeitschriften zum Ausdruck. All diese Beurteilungen benutzen Kennzahlen, die nicht kritisch untersucht werden. gewissen Zeitraum vermittelten Absolventen und die Einstiegsgehälter anbieten. Es soll hier nicht untersucht werden, welche Schwierigkeiten schon bei dieser einfachen Kennzahl in der praktischen Umsetzung auftreten können. Auch andere Kriterien müssen sich aus der Kundenzufriedenheit ableiten lassen. Hierfür könnten bereits etablierte Methoden der Messbarkeit für Kundenzufriedenheit benutzt werden.

Wie beurteilt man den Studenten mit seiner Vorbildung und seiner Grundeinstellung? Während man die Vorbildung relativ leicht messen kann, beispielsweise durch eine Anfangsüberprüfung in Form einer Klausur, ist die Grundeinstellung, die auch eng mit der Motivation verbunden ist, wesentlich schwieriger zu messen. Eine Befragung wäre eine Möglichkeit, um Ergebnisse zu bekommen. Es ist aber allgemein bekannt, dass sich eine optimale Einstellung zum Studium erst im Laufe der ersten Semester herausbildet. Ein anderes gravierendes Problem ist das Auseinanderklaffen des Anspruches und der Leistungsbereitschaft. Dies belegen Studien über die Evaluierung an Hochschulen. Somit sind Befragungen als Messinstrument sehr komplex und schwierig zu gestalten, um den hohen subjektiven Anteil zu begrenzen. Werden diese Befragungen nicht sorgfältig genug durchgeführt, so sind die erhaltenen Ergebnisse nicht einmal hochschulintern und erst recht nicht in einem größeren Zusammenhang interpretierbar. Sie sind schlicht bedeutungslos für eine Evaluation. Sie können nur als eine Meinungsäußerung gewertet werden. In einer Evaluation bei Befragung der Studenten ist das Semester als objektive Zeitvariable und die mit der Studiendauer veränderliche Grundeinstellung zu berücksichtigen, die auch mit dem persönlichen Studienerfolg eng verbunden ist.

\section{Evaluationsgrundsätze}

1. Vergleiche können nur bei objektivierender Analyse durchgeführt werden.

2. Objektivierte hochschulinterne Analysen können der strukturellen und methodischen Verbesserung dienen. Sie können, falls sie auf der gleichen Grundlage durchgefuihrt werden, zum strukturellen und methodischen Vergleich zwischen den Hochschulen genutzt werden. Keinesfalls rechtfertigen sie ein Ranking.

3. Objektive externe Beurteilungen durch die Kunden (Wirtschaft, Verwaltungen und gesellschaftliche Institutionen) bilden die Grundlage für ein sinnvolles, den Entwicklungsprozess der Hochschulen förderndes Ranking.

Es war die Absicht, einen Problemüberblick zu geben. Die Ausarbeitung einer Evaluationsvorschrift für die Hochschule Wildau wird einem anderen Artikel vorbehalten bleiben. Auch eine vollständige Literaturauswertung wurde nicht angestrebt. Vielmehr soll die Diskussion angeregt werden. 


\section{Anmerkungen}

[1] vgl. Heike Solga, Differenzierte Hochschulen, Ein Plädoyer für mehr Effizienz und Durchlässigkeit, in: Aus Politik und Zeitgeschichte, B 15/98, S. 20-29

[2] vgl. Winfried Schlaffke/Christiane Konegen-Grenier, Hochschulreform aus Sicht der Wirtschaft, in: Aus Politik und Zeitgeschichte, B 15/98, S. $31 \mathrm{ff}$.

[3] vgl. F. H. Rüger, Studienrefom im Widerstreit von Anglophilie und Anglophobie, in: Semesterjournal der Fachhochschule für Wirtschaft Berlin, Nov. 1998

[4] „Studenten prüfen ihre Universitäten“, FAZ vom 05. 02. 1999

[5] vgl. Europäische Pilotprojekte für die Qualitätsbewertung im Bereich der Hochschulen, Bundesrepublik Deutschland. Nationaler Bericht, Dokumente zur Hochschulreform 105/1995, S. 7 ff.

[6] ebenda, S. 21

[7] ebenda, S. 23

[8] ebenda, S. 25

[9] Werner Stangl, Die Evaluation universitärer Lehrveranstaltungen, Universität Linz, Internet, 14.04.1999, S. 3

[10] vgl. Winfried Schlaffke/Christiane Konegen-Grenier, a.a.O. S. 36

[11] Natali el Hage, Lehrevaluation und studentische Veranstaltungskritik. Projekte, Instrumente und Grundlagen, Bundesministerium für Bildung, Wissenschaft, Forschung und Technologie, Bonn 1997, S. 140

\section{Autor}

\section{Prof. Dr. Peter Gadow}

Technische Fachhochschule Wildau

Fachbereich Wirtschaft, Verwaltung und Recht

Tel. (0 33 75) 508-506

E-Mail: pgadow@wvr.tfh-wildau.de 